\title{
Direct Correlation between Proliferative Activity and Dysplasia in Pancreatic Intraepithelial Neoplasia (PanIN): Additional Evidence for a Recently Proposed Model of Progression
}

Walter M. Klein, M.D., Ralph H. Hruban, M.D., Andres J.P. Klein-Szanto, M.D., Robb E. Wilentz, M.D.

Departments of Pathology (WMK, RHH, REW) and Oncology (RHH), The Johns Hopkins Medical

Institutions, Baltimore, Maryland, and the Department of Pathology (AJPK-Z), Fox Chase Cancer Center, Philadelphia, Pennsylvania

A growing body of morphological, clinical, and genetic observations suggests a progression model for pancreatic ductal adenocarcinoma. In this model, pancreatic ducts progress through a series of architectural and cytological changes that define degrees of pancreatic intraepithelial neoplasia (PanIN). Expressed in dividing cells, Ki-67 has been extensively used as a proliferation marker. Its expression in different grades of PanIN has not been well studied. A total of 76 PanINs from 41 patients were histologically graded according to recently established criteria. These PanINs were then immunolabeled with a monoclonal antibody against Ki-67 (Mib-1). Normal ducts and invasive ductal adenocarcinomas were also labeled with the antibody. In 15 normal ducts, only $0.41 \%$ of the epithelial cells expressed Ki-67. Ki-67-labeling indices in the increasing grades of PanIN were as follows: PanIN-1A, 0.69\%; PanIN-1B, 2.33\%; PanIN-2, 14.08\%; and PanIN-3, 22.01\%. Fifteen invasive ductal adenonocarcinomas showed an average labeling index of $\mathbf{3 6 . 9 9 \%}$. The difference in Ki-67 labeling among these groups was statistically significant $(P<.0005$, Kruskal-Wallis test). This pattern of proliferation provides additional evidence supporting the recently proposed pancreatic progression model. It also correlates well with known molecular changes, such as activating

Copyright () 2002 by The United States and Canadian Academy of Pathology, Inc.

VOL. 15, NO. 4, P. 441, 2002 Printed in the U.S.A

Date of acceptance: October 29, 2001.

Supported in part by the Specialized Program of Research Excellence (SPORE) in Gastrointestinal Cancer, P50-CA62924.

Presented in part at the United States and Canadian Academy of Pathology meeting in Atlanta, Georgia, March, 2001.

Address reprint requests to: Robb E. Wilentz, M.D., Ross Building 632, 720

Rutland Avenue, Department of Pathology, The Johns Hopkins Hospital,

Baltimore, MD 21205; e-mail: rwilentz@jhmi.edu; fax: 410-614-0671. point mutations in the $\mathrm{K}$-ras oncogene and the loss of DPC4 and p16 gene expression. Ki-67 staining may be useful as an adjunct in the diagnosis of precancerous lesions in the pancreas and may provide a reliable way to identify lesions at high risk for the subsequent development of infiltrating carcinoma.

KEY WORDS: Ki-67 Pancreas, Pancreatic Intraepithelial Neoplasia, PanIN, Proliferation.

Mod Pathol 2002;15(4):441-447

Well-defined noninvasive lesions are often found in association with infiltrating pancreatic ductal adenocarcinoma. These Tesions, together known as pancreatic intraepithelial neoplasia (PanIN), have been recently classified at a National Cancer Institute-sponsored Pancreas Cancer Think Tank (1). PanINs are thought to progress from flat and papillary lesions without dysplasia, to papillary lesions with dysplasia, to lesions showing carcinoma in situ (PanIN-1A to PanIN1-B to PanIN-2 to PanIN-3). Table 1 and Figure 1 provide descriptions of these grades of PanIN. (A more thorough discussion of PanINs can be found at www.pathology.jhu.edu/pancreas_panin).

The genetic alterations present in PanINs have been extensively studied. PanINs have been examined for loss of heterozygosity at a number of loci and for alterations in several genes such as K-ras, p16, p53, DPC4, and BRCA2 (2-10). These genetic studies, along with histologic observations, support a progression model for pancreatic neoplasia in which increasing histologic grades of PanIN are associated with the accumulation of genetic alterations in cancer-associated genes (Fig. 1) (11).

The validity of this model is now under study. One way to test this model's applicability is to study the proliferation rate of the epithelial cells in the 
TABLE 1. Labeling Index of Pancreatic Ductal Lesions, Normal Ductal Epithelium, and Infiltrating Ductal Adenocarcinoma

\begin{tabular}{|c|c|c|c|c|c|c|c|}
\hline Group & Pathologic Description & $N$ & Mean (\%) & Median (\%) & $\operatorname{Min}(\%)$ & $\operatorname{Max}(\%)$ & SD \\
\hline Infiltrating & $\begin{array}{l}\text { Invasive glands within a } \\
\text { desmoplastic stroma }\end{array}$ & 15 & 36.99 & 35.10 & 23.93 & 53.27 & 9.06 \\
\hline Normal & $\begin{array}{l}\text { Nonmucinous flattened or } \\
\text { cuboidal epithelium without } \\
\text { dysplasia }\end{array}$ & 15 & 0.41 & 0.49 & 0 & 1.02 & 0.34 \\
\hline PanIN-1A & $\begin{array}{l}\text { Flat, mucinous epithelium } \\
\text { without dysplasia }\end{array}$ & 20 & 0.69 & 0.46 & 0 & 3.70 & 0.87 \\
\hline PanIN-1B & $\begin{array}{l}\text { Papillary, mucinous epithelium } \\
\text { without dysplasia }\end{array}$ & 20 & 2.33 & 1.92 & 0.52 & 10.60 & 2.21 \\
\hline PanIN-2 & $\begin{array}{l}\text { Flat or papillary, mucinous } \\
\text { epithelium with mild-to- } \\
\text { moderate dysplasia (mild-to- } \\
\text { moderate nuclear irregularity, } \\
\text { hyperchromasia, and loss of } \\
\text { polarity) }\end{array}$ & 18 & 14.08 & 12.59 & 3.82 & 28.63 & 7.76 \\
\hline PanIN-3 & $\begin{array}{l}\text { Flat or papillary, mucinous } \\
\text { epithelium with severe } \\
\text { dysplasia (marked nuclear } \\
\text { irregularity, hyperchromasia, } \\
\text { and loss of polarity), often with } \\
\text { cribriforming and intraluminal } \\
\text { "blebbing" }\end{array}$ & 18 & 22.01 & 21.73 & 5.76 & 38.94 & 9.77 \\
\hline
\end{tabular}

Infiltrating, infiltrating ductal adenocarcinoma; Min, minimum; Max, maximum.

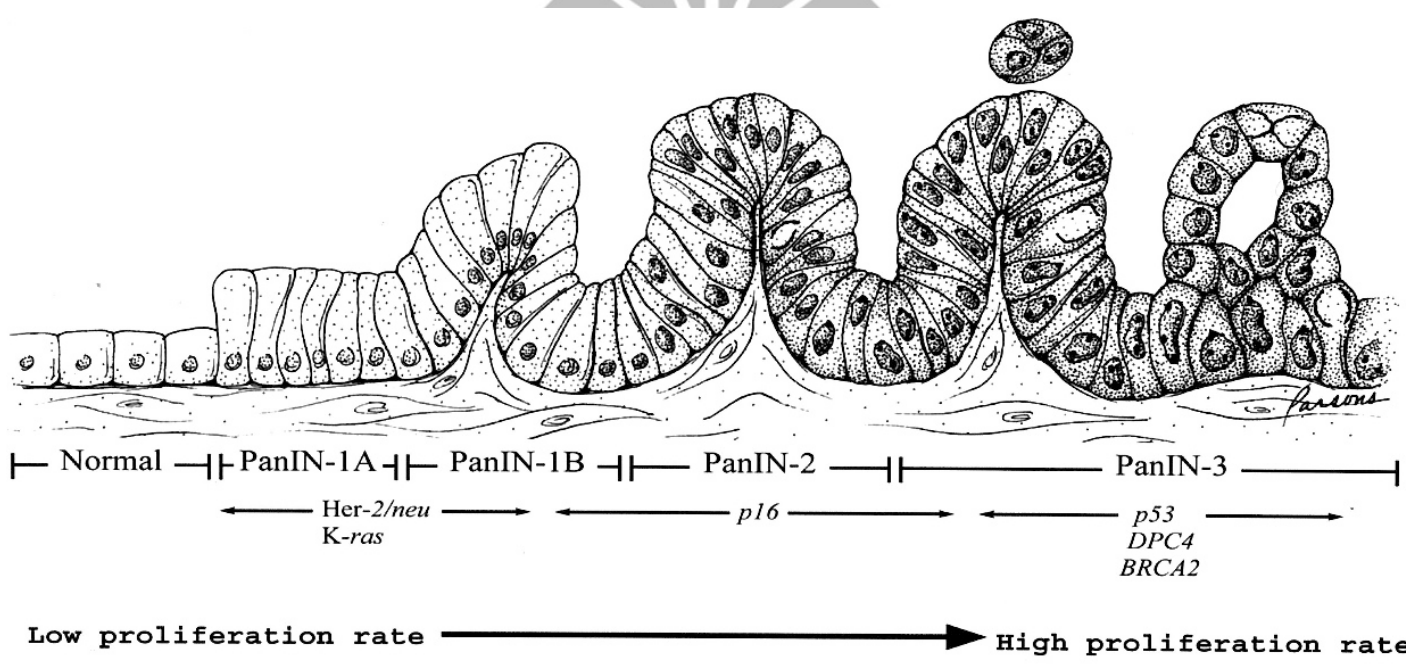

\section{Progression Model of Pancreatic Cancer}

FIGURE 1. Histologic-genetic progression model for pancreatic cancer. Adapted with permission from Wilentz et al. (10). Artwork by Jennifer Brumbaugh.

successive grades of PanIN. We would anticipate that, as is true in other organs, the proliferation rate should correlate with increasing grades of dysplasia.

Proliferation rate can be determined by labeling a large number of PanINs with an antibody against Ki-67. Ki-67 is a nuclear protein that correlates with cellular proliferation (12). Many studies have investigated the relationship of Ki-67 labeling with prognosis in a variety of different types of neoplasia. Although Ki-67 expression has been studied in some pancreatic lesions (13), it has not been evaluated using the currently accepted nomenclature and diagnostic criteria.
Therefore, we examined the expression of Ki-67 in a large panel of PanINs. We demonstrate that the Ki-67 index directly parallels the morphologic findings in PanINs, providing additional evidence for the pancreatic progression model.

\section{MATERIALS AND METHODS}

\section{Specimen Selection}

Pancreaticoduodenectomy specimens from 41 patients were studied. Thirty-nine resections were 
performed for infiltrating carcinoma, and 2 resections were performed for chronic pancreatitis.

\section{Identification of Duct Lesions}

Multiple hematoxylin and eosin-stained slides of pancreatic tissue from each of the cases were screened by light microscopy for PanINs simultaneously by two of the coauthors (WMK, REW). Each PanIN was graded according to criteria established at the National Cancer Institute-sponsored Pancreas Cancer Think Tank (September, 1999, Park City, UT; 1). Briefly, PanINs-1A and -1B respectively showed flat or papillary mucinous epithelium without dysplasia, whereas PanINs-2 had flat or papillary mucinous epithelium with mild-to-moderate dysplasia (mild-to-moderate nuclear irregularity, hyperchromasia, and loss of polarity). PanINs-3 contained severe dysplasia (marked nuclear irregularity, hyperchromasia, and loss of polarity) and were often associated with cribriforming structures and/or intraluminal blebbing. (See Fig. 1, Table 1, and www.pathology.jhu.edu/pancreas_panin).

Agreement between the observers with respect to grading of the PanIN lesions was highly robust, with disagreement noted in only a minority of cases $(5 / 76,6.6 \%)$. The disagreement between the two observers was never more than one grade of PanIN (e.g., PanIN-1B versus PanIN-2). This disagreement was resolved after discussion at the microscope.

The slides were derived from a total of 58 separate blocks. Unstained $5-\mu \mathrm{m}$ sections containing the PanINs were then cut from these paraffin blocks for subsequent immunohistochemical analysis.

\section{Immunohistochemistry}

Unstained sections were treated with a monoclonal antibody against Ki-67 at a dilution of 1:100 (Mib-1, mouse monoclonal, Immunotech, Westbrook, ME). Antigen retrieval was performed for 5 minutes in $0.01 \mathrm{M}$ sodium citrate buffer. An avidin-biotin-peroxidase kit (Vectastain Elite, Burlingame, CA) was employed, and 3'3'-diaminobenzidine (3,3'-diaminobenzidine) was used to develop the immunostain. Sections without the primary antibody were used as negative controls. All sections were counterstained with hematoxylin and mounted.

\section{Labeling Index and Statistical Analysis}

Assessment of ductal cells labeled with Ki-67 was done by light microscopy under a $40 \times$ objective by one of the coauthors (WMK). Cells were generally uniformly distributed within each neoplastic lesion. For each PanIN, randomly selected high-power fields were studied until either the entire duct was analyzed or a minimum of 500 cells was counted. Cells with a visible, granular nuclear reaction that was brownish in color were regarded as positively labeled. The cells within germinal centers of adjacent lymph nodes served as internal positive controls. For each PanIN, the labeling index (positive cells/total cells $\times 100$ ) was determined.

Differences in Ki-67 labeling among normal ducts, PanIN-1A, PanIN-1B, PanIN-2, PanIN-3, and infiltrating adenocarcinoma were assessed using the KruskalWallis test. Bonferroni-corrected Mann-Whitney tests were used to make all pairwise comparisons among the six groups. The Type I error rate was kept below the nominal $5 \%$ level.

\section{RESULTS}

\section{Normal Ducts and Associated Infiltrating Adenocarcinoma}

Seventy-six PanINs, 15 normal pancreatic ducts, and 15 associated infiltrating ductal adenocarcinomas were examined from the 41 pancreata included in this study. Of the 41 pancreata, 33 had been removed for conventional pancreatic ductal adenocarcinoma, 5 for cholangiocarcinoma, 1 for metastatic renal cell carcinoma, and 2 for chronic pancreatitis.

The 15 normal ducts had a labeling index of $0.41 \%$. In contrast, the 15 cases with infiltrating adenocarcinoma had an average labeling index of $36,99 \%$. These two values served as reference points for comparing the Ki-67 staining indices in PanINs. Figure 2 shows the high labeling index of an infiltrating adenocarcinoma, compared with that in an adjacent/normal duct/PanIN-1A.

\section{PanINs}

Twenty PanINs-1A, 20 PanINs-1B, 18 PanINs-2, and 18 PanINs-3 were identified within the 41 pancreata. In PanIN-1A, very few ductal epithelial cells labeled (labeling index of $0.69 \%$ ). The PanINs-1B had a slightly higher labeling index of $2.33 \%$. In contrast, the labeling indices within PanINs-2 and -3 were markedly increased $(14.08 \%$ and $22.01 \%$, respectively). Figure 3 demonstrates the increasing degree of immunolabeling observed in four representative PanINs. This increase in immunolabeling along the successive stages of PanIN was statistically significant $(P<.0005$, Kruskal-Wallis test). Figure 4 presents a graphical comparison of the labeling indices among normal ducts, PanINs, and infiltrating adenocarcinomas.

\section{Quantitative Analysis}

The descriptive statistics for the Ki-67 labeling indices are provided in Table 1. In summary, a Kruskal-Wallis test indicated that there were statistically significant differences $(P<.0005)$ among the 

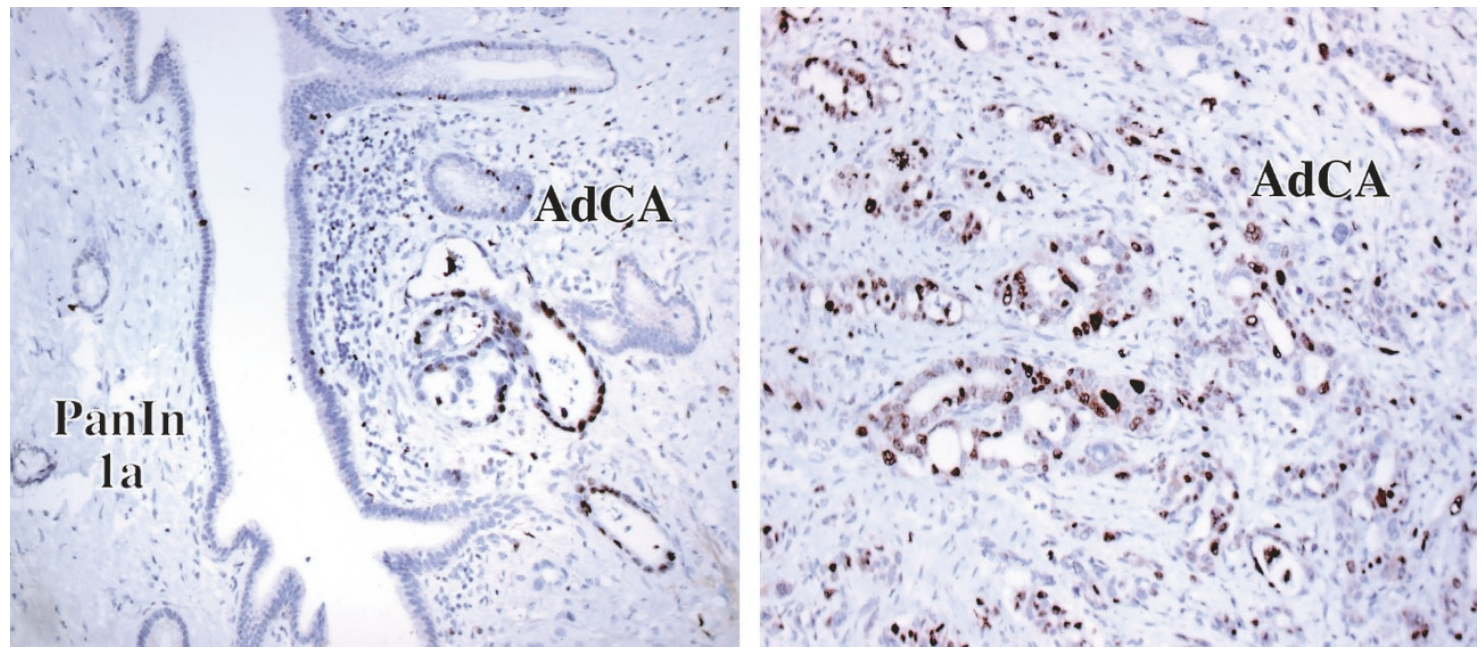

FIGURE 2. Ki-67-labeling indices in normal duct/PanIN-1A and infiltrating ductal adenocarcinoma. The panel on the left shows a comparison of labeling between infiltrating adenocarcinoma (AdCA, high labeling) and normal duct/PanIN-1A (low labeling). The panel on the right contains a high-power view of infiltrating adenocarcinoma, in which approximately one third of the nuclei stain.
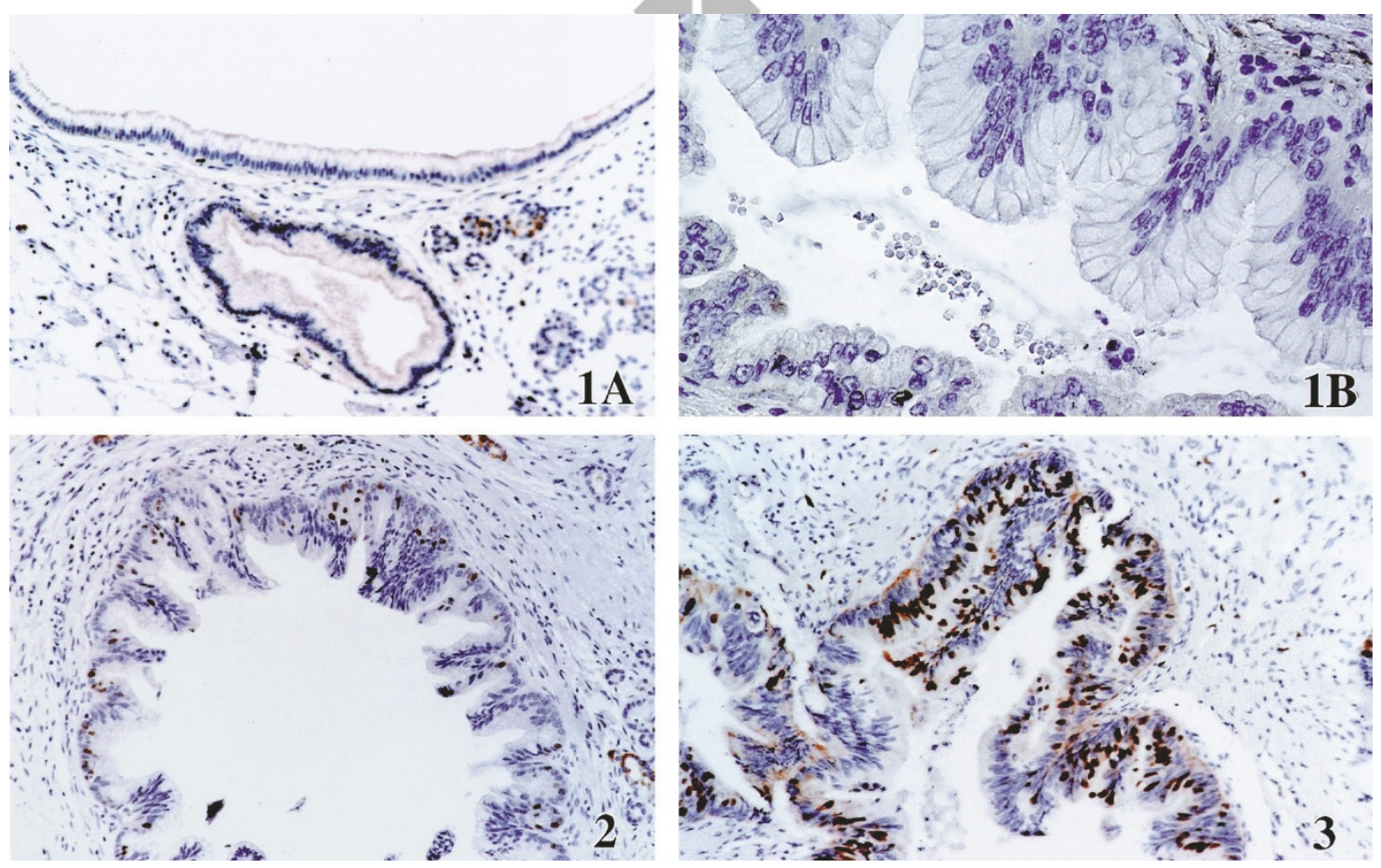

FIGURE 3. A comparison of Ki-67 labeling indices among the successive stages of PanIN. As can be seen in this figure, PanINs-2 and -3 show significantly increased labeling indices as compared with PanIN-1.

six groups. More specifically, this analysis also showed that there were no significant differences in the Ki-67-labeling indices between the normal duct (Nl) and PanIN-1A groups. The labeling of the $\mathrm{Nl}$ and PanIN-1A groups was significantly lower than the labeling indices of the PanIN-1B, PanIN-2, PanIN-3, and infiltrating adenocarcinoma groups. In addition, the labeling index of the PanIN-1B group was significantly lower than those of the PanIN- 2, PanIN-3, and infiltrating adenocarcinoma groups. There were no significant differences in the labeling indices between the PanIN-2 and
PanIN-3 groups. Nevertheless, PanIN-2 and PanIN-3 had labeling indices that were significantly lower than that of the infiltrating adenocarcinoma group (Table 1).

\section{DISCUSSION}

The proliferation rates in malignant tumors and their precursor lesions have been studied for several decades. Ki-67 labeling has gradually supplanted other techniques previously used to evalu- 


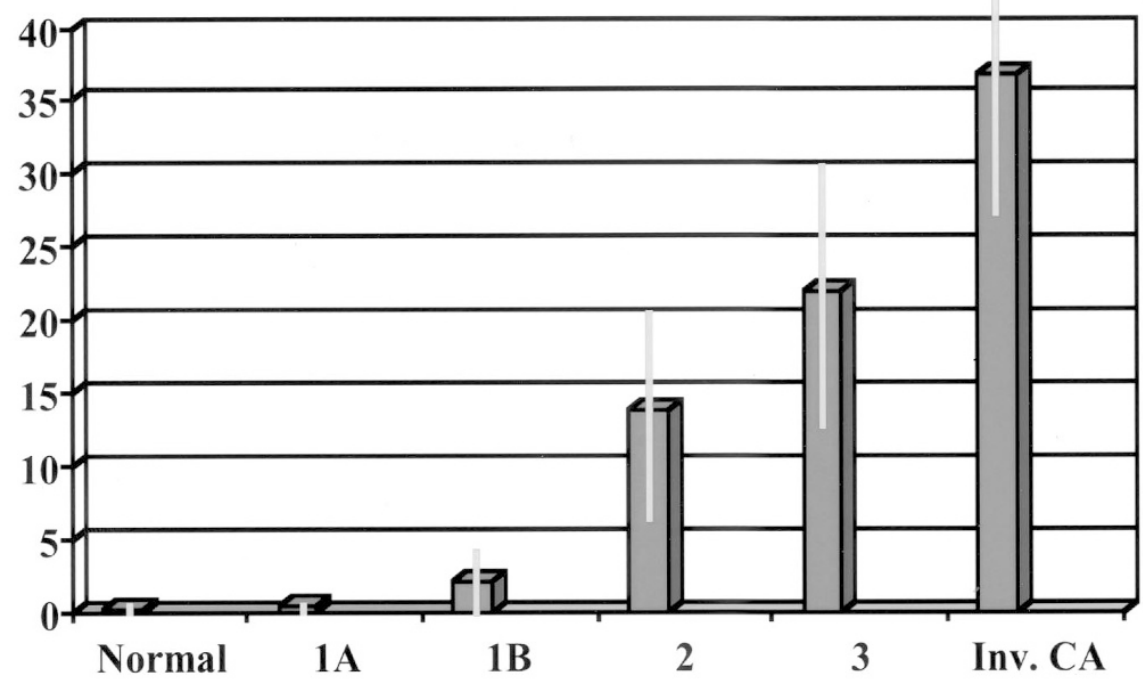

Ki-67 LI

FIGURE 4. Histogram showing the Ki-67 labeling indices in PanINs, compared with normal ducts and infiltrating ductal adenocarcinoma. Proliferative activity increases in parallel with increasing grade of dysplasia.

ate proliferation rates in neoplasias. Indeed, Ki-67 expression correlates with other parameters of cell proliferation, such as thymidine labeling index, S-phase fraction, and mitotic count (14).

The Ki-67 monoclonal antibody detects a nonhistone protein of unknown function that is present in all phases of the cell cycle, except G0 and very early G1. Although the original Ki-67 antibody was reâctive only in frozen sections, a newer antibody to an epitope of Ki-67 (Mib-1) is active in formalin-fixed, paraffin-embedded tissues. It has therefore been used extensively to evaluate/malignancies in many different organ sites (15-22).

Ki-67 labeling with Mib-1 has been used to study not only invasive lesions but also the precursors to these invasive lesions. For example, several studies have analyzed the proliferative activity of squamous intraepithelial lesions in the cervix and head and neck (15-18). The precursors to infiltrating adenocarcinomas have likewise been explored (19-22). In general, proliferation has been directly proportional to the degree of dysplasia, in other words, the grade of the precursor intraepithelial lesion. For instance, carcinomas in situ have a higher labeling index than lesions with only mild dysplasia.

Although Ki-67 labeling has been used to study neoplastic progression in many organ systems, it has not been used extensively within the pancreas. One neoplasm that has been studied within the pancreas is the intraductal papillary mucinous neoplasm (IPMN), which can give rise to infiltrating adenocarcinoma (23). However, most infiltrating duct adenocarcinomas of the pancreas do not derive from IPMNs. Indeed, a growing body of evidence suggests that most pancreatic ductal adenocarcinomas arise from microscopic precursor lesions called PanINs. These PanINs can progress from flat duct lesion (PanIN-1A), to papillary duct lesion without dysplasia (PanIN-1B), to papillary duct lesion with dysplasia (PanIN-2), to carcinoma in situ (PanIN-3), and finally to infiltrating ductal adenocarcinoma (Fig. 1).

This model is based on three lines of evidencemorphological, clinical, and genetic (24). Morphologically, pancreatic duct lesions have been shown to be three times more common in pancreata from patients with infiltrating carcinoma than they are in pancreata from patients without carcinoma (25, 26). Moreover, three-dimensional mapping techniques have demonstrated a stepwise progression from mild dysplasia to severe dysplasia in duct lesions (27). Clinically, several reports have established the temporal progression of pancreatic duct lesions to infiltrating adenocarcinoma $(28,29)$.

Perhaps the strongest evidence for the recently proposed model of pancreatic cancer progression, however, is genetic. Pancreatic intraepithelial neoplasias (PanINs) contain many of the same genetic changes that are seen in infiltrating pancreatic adenocarcinoma. For example, alterations in the $K$-ras, p16, BRCA2, p53, and DPC4 genes have been detected in PanINs (2-10). Not surprisingly, the prevalence of many of these alterations increases with the increasing grade of the PanIN.

The recent acceptance of an international nomenclature and diagnostic criteria for precursor lesions in the pancreas provides the opportunity to study proliferation rates in the various lesions in this new grading system. We therefore examined the expression of the Ki-67 protein in a spectrum of PanINs to seek additional evidence for the recently proposed model of pancreatic cancer progression. 
Ki-67 immunolabeling was carefully correlated with histology using the recently adopted PanIN nomenclature and grading scheme (1). Although a previous study examined proliferative activity within pancreatic precursor lesions, it did not use the newly adopted PanIN nomenclature (13). The goal of this study was to determine whether the Ki-67-labeling rate paralleled an increasing grade of dysplasia in this newly adopted model in order to provide evidence for or against the new model.

Our study examined PanINs in 33 patients with infiltrating pancreatic ductal adenocarcinoma, 5 with infiltrating cholangiocarcinoma, 1 with a metastatic renal cell carcinoma, and 2 with chronic pancreatitis. Similar to precursor lesions in other organ systems, PanINs showed a statistically significant increase in proliferative rate in parallel with increasing stages of dysplasia (Fig. 4). The lack of a significant difference in the Ki-67-labeling index between PanIN-2 and PanIN-3 may represent the limited statistical power of this study. More cases will be necessary to determine whether the difference in labeling between PanIN-2 and PanIN-3 is real.

These data support the new model and nomenclature for precursor lesions in the pancreas. As new molecular findings emerge, the classification of PanIN will serve as a framework for the interpretation of these findings. Furthermore, it will be of interest to determine whether any of the identified genetic alterations can account for the increasing proliferation rates identified in this study (30). In addition, it is possible that Ki-67 labeling indices may serve as an alternate or back-up grading system for PanINs, that Ki-67 labeling may be a reliable marker for the biologic risk of a lesion, and that Ki-67 labeling may be an accurate way to assess high-risk precursor lesions shed into pancreatic secretions. Additional genetic, clinical, and immunohistochemical data, including those in reactive ducts and ducts with squamous metaplasia, will be needed to explore these areas. These data will lead to a better understanding of the pancreatic progression model. This in turn may result in new diagnostic modalities to help identify patients at risk for the nearly universally fatal disease of pancreatic cancer.

Acknowledgments: We would like to thank James Babb (Biostatistics Facility, Fox Chase Cancer Center) for assistance with the statistical analyses.

\section{REFERENCES}

1. Hruban RH, Volkan NA, Albores-Saavedra J, Compton C, Garrett ES, Goodman SN, et al. Pancreatic intraepithelial neoplasia: a new nomenclature and classification system for pancreatic duct lesions. Am J Surg Pathol 2001;25:579-86.
2. Moskaluk CA, Hruban RH, Kern SE. p16 and K-ras gene mutations in the intraductal precursors of human pancreatic adenocarcinoma. Cancer Res 1997;57:2140-3.

3. Wilentz RE, Geradts J, Maynard R, Offerhaus GJA, Kang M, Goggins $\mathrm{M}$, et al. Inactivation of the p16 (INK4A) tumor suppressor gene in pancreatic duct lesions: loss of intranuclear expression. Cancer Res 1998;58:4740-4.

4. DiGiuseppe JA, Hruban RH, Goodman SN, Polak M, van den Berg FM, Allison DC, et al. Overexpression of the p53 protein in adenocarcinoma of the pancreas. Am J Clin Pathol 1994; 101:684-8.

5. Day JD, DiGiuseppe JA, Yeo CJ, Loi-Goldman M, Anderson S, Kern SE, et al. Immunohistochemical evaluation of HER-2/ neu oncogene expression in pancreatic adenocarcinoma and pancreatic intraepithelial neoplasms. Hum Pathol 1996; 27:119-24.

6. Yanagisawa A, Ohtake K, Ohashi K, Hori M, Kitagawa T, Sugano $\mathrm{H}$, et al. Frequent c-Ki-ras oncogene activation in mucous cell hyperplasias of pancreas suffering from chronic inflammation. Cancer Res 1993;53:953-6.

7. Tada M, Ohashi M, Shiratori Y, Okudaira T, Komatsu Y, Kawabe T, et al. Analysis of K-ras gene mutation in hyperplastic duct cells of the pancreas without pancreatic disease. Gastroenterology 1996;110:227-31.

8. Caldas C, Hahn SA, Hruban RH, Redston MS, Yeo CJ, Kern SE. Detection of K-ras mutations in the stool of patients with pancreatic adenocarcinoma and pancreatic ductal hyperplasia. Cancer Res 1994;54:3568-73.

9. Hameed M, Marrero AM, Conlon KC, Brennan MF, Klimstra DS. Expression of p53 nucleophosphoprotein in in situ pancreatic ductal adenocarcinoma: an immunohistochemical analysis of 100 cases [abstract]. Lab Invest 1994;70:132A.

10. Wilentz RE, Iacobuzio-Donahue CA, Argani P, McCarthy DM, Parsons JL, Yeo CJ, et al. Loss of expression of Dpc4 in pancreatic intraepithelial neoplasia: evidence that DPC4 inactivation occurs late in neoplastic progression. Cancer Res 2000;60:2002-6.

11. Hruban RH, Wilentz RE, Kern SE. Genetic progression in pancreatic ducts. Am J Pathol 2000;156:1821-5.

12. Scholzen T, Gerdes J. The Ki-67 protein: from the known and unknown. J Cell Physiol 2000;182:311-22.

13. Tomaszewska R, Okon K, Nowak K, Stachura J. Proliferation index and karyometric features of pancreatic intraductal proliferative lesions. Anal Cell Pathol 1999;19:175-85.

14. Keshgegian AA, Anaan A. Proliferation markers in breast carcinoma: mitotic figure count, s-phase fraction, proliferating cell nuclear antigen, Ki-67 and Mib-1. Am J Clin Pathol 1995;104:42-9.

15. Bulten J, van der Laak JA, Gemmink JH, Pahlplatz MM, de Wilde PC, Hanselaar AG. MIB1, a promising marker for the classification of cervical intraepithelial neoplasia. J Pathol 1996;178:268-73.

16. Isacson C, Kessis TD, Hedrick L, Cho KR. Both cell proliferation and apoptosis increase with lesion grade in cervical neoplasia but do not correlate with human papillomavirus type. Cancer Res 1996;56:669-74.

17. Kushner J, Bradley G, Jordan RCK. Patterns of p53 and Ki-67 protein expression in epithelial dysplasia from the floor of the mouth. J Pathol 1997;183:418-23.

18. Liu SC, Sauter ER, Clapper ML, Feldman RS, Levin L, Chen SY, et al. Markers of cell proliferation in normal epithelia and dysplastic leukoplakias of the oral cavity. Cancer Epidemiol Biomarkers Prev 1998;7:597-603.

19. Shpitz B, Bomstein Y, Mekori Y, Cohen R, Kaufman Z, Grankin M, et al. Proliferating cell nuclear antigen as a marker of cell kinetics in aberrant crypt foci, hyperplastic polyps, adenomas, and adenocarcinomas of the human colon. Am J Surg 1997;174:425-30. 
20. Whittles CE, Biddlestone LR, Burton A, Barr H, Jankowski JAZ, Warner PJ, et al. Apoptotic and proliferative activity in the neoplastic progression of Barrett's oesophagus: a comparative study. J Pathol 1999;187:535-40.

21. Yang SY, Liu SC, Al-Saleem LF, Holloran D, Babb J, Guo X, et al. E2F-1: a proliferative marker of breast neoplasia. Cancer Epidemiol Biomarkers Prev 2000;9:395-401.

22. Tamboli P, Amin MB, Schultz DS, Linden MD, Kubus J. Comparative analysis of the nuclear proliferative index (Ki-67) in benign prostate, prostatic intraepithelial neoplasia, and prostatic carcinoma. Mod Pathol 1996;9:1015-9.

23. Terada T, Ohta T, Kitamura Y, Ashida K, Matsunga Y. Cell proliferative activity in intraductal papillary-mucinous neoplasms and invasive ductal adenocarcinomas of the pancreas: an immunohistochemical study. Arch Pathol Lab Med 1998;122:42-6.

24. Hruban RH, Goggins M, Parsons J, Kern SE. Progression model for pancreatic cancer. Clin Cancer Res 2000;6:296972.

25. Cubilla A, Fitzgerald PJ. Morphological lesions associated with human primary invasive nonendocrine pancreas cancer. Cancer Res 1976;36:2690-8.
26. Kozuka S, Sassa R, Taki T, Masamoto K, Nagasawa S, Saga S, et al. Relation of pancreatic duct hyperplasia to carcinoma. Cancer 1979;43:1418-28.

27. Furukawa T, Chiba R, Kobari M, Matsuno S, Nagura H, Takahashi T. Varying grades of epithelial atypia in the pancreatic ducts of humans. Classification based on morphometry and multivariate analysis and correlated with positive reactions of carcinoembryonic antigen. Arch Pathol Lab Med 1994;118:227-34.

28. Brat DJ, Lillemoe KD, Yeo CJ, Warfield PB, Hruban RH. Progression of pancreatic intraductal neoplasias (high-grade PanIN) to infiltrating adenocarcinoma of the pancreas. Am J Surg Pathol 1998;22:163-9.

29. Brockie E, Anand A, Albores-Saavedra J. Progression of atypical ductal hyperplasia/carcinoma in situ of the pancreas to invasive adenocarcinoma. Ann Diagn Pathol 1998;2:286-92.

30. Luttges J, Galehdari H, Brocker V, Schwarte-Waldhoff I, Henne-Burns D, Kloppel G, et al. Allelic loss is often the first hit in the biallelic inactivation of the p53 and DPC4 genes during pancreatic carcinogenesis. Am J Pathol 2001; 158:1677-83.

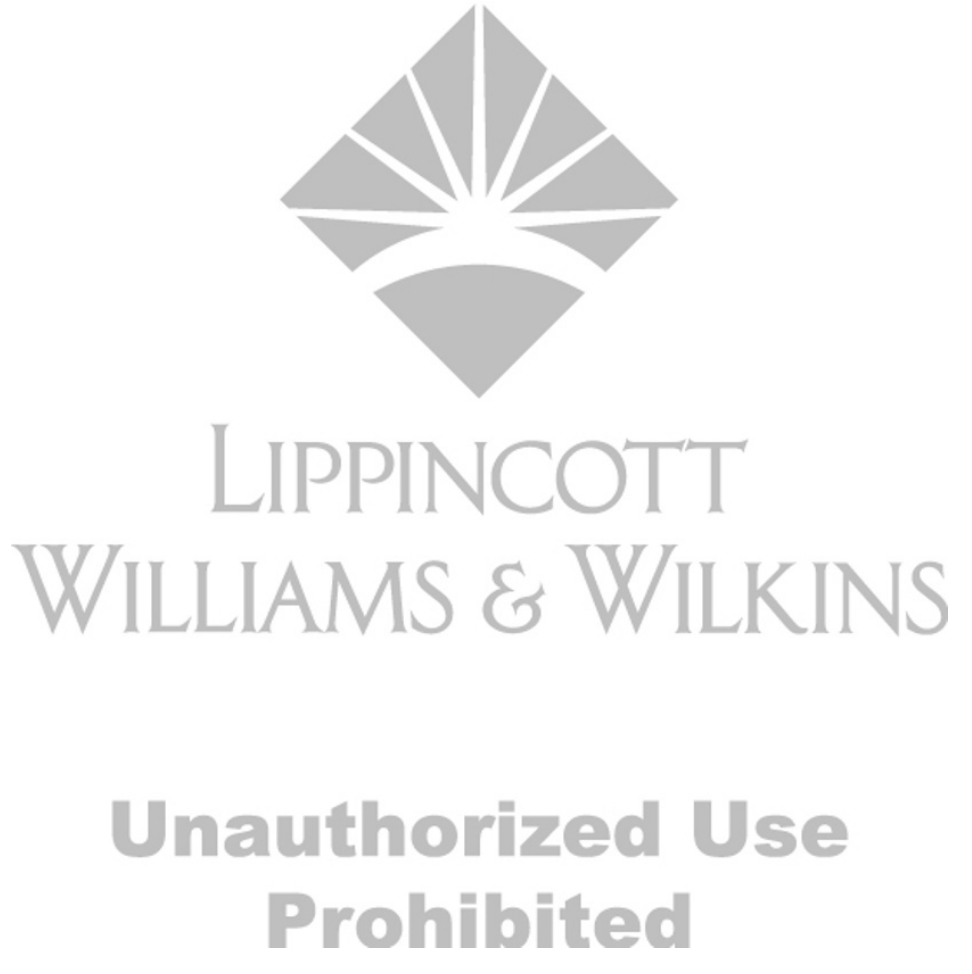

